\title{
Interaction between viral particles and structured metal surface under surface plasmon propagation
}

\author{
V. Lozovski ${ }^{1,2}$, V. Lysenko ${ }^{1}$, M. Spivak ${ }^{3}$, V. Sterligov ${ }^{1}$ \\ ${ }^{1} V$. Lashkaryov Institute of Semiconductor Physics, NAS of Ukraine, \\ 45, prospect Nauky, 03028 Kyiv, Ukraine \\ ${ }^{2}$ Institute of High Technologies, Taras Shevchenko Kyiv National University, \\ 64, Volodymyrska str., 01601 Kyiv, Ukraine \\ ${ }^{3}$ D. Zabolontyi Institute of Microbiology and Virology, NAS of Ukraine, \\ 154, Zabolotnyi str., 13143 Kyiv, Ukraine
}

\begin{abstract}
The new method for decontamination of biological liquids (blood or blood plasma, for instance) from viruses is proposed. The method is based on the efficient attraction between nanoparticles and nanostructured surface, under which the surface plasmon-polaritons propagate.
\end{abstract}

Keywords: nanoparticle, virus, local-field enhancement, surface plasmon-polariton.

Manuscript received 29.12.11; revised version received 16.01.12; accepted for publication 26.01.12; published online 29.03.12.

\section{Introduction}

A virus is a small "semi-living" particle that is infectious agent able to replicate only inside living cells of organisms [1,2]. The struggle against viruses has become up to date the most important problem of the living sciences. The characteristic dimensions of viruses are about few tens of nanometers. For example, the dimensions of adenoviruses are close to $70 \ldots 90 \mathrm{~nm}$, the dimension of AIDS virus reaches $100 \ldots 120 \mathrm{~nm}$, and dimensions of herpes viruses are approximately $170 \mathrm{~nm}$. It means that the viruses are the objects of nanophysics, specifically, near-field physics [3-5]. As viruses are organic supramolecular structure, from the electrodynamics viewpoint it is characterized both by linear and nonlinear responses. Then, it should actively interact with the surfaces, especially, with the nanostructured ones. As it is well known [6], interaction between nanoparticles and nanostructured surfaces is related with local fields. The effect of local-field enhancement is a characteristic phenomena of nanooptics [7]. The local-field enhancement can, for example, lead to amplification of interaction between the surface and the nanoparticle. This amplification can be observed for nanoparticles with dimensions close to characteristic linear dimensions of nanostructures at the surface. Then, the possibility for selective adsorption of nanoparticles (with the above linear dimensions) arises. This possibility is the main idea of using the nanostructured surface under special condition to rectify biological liquids (for example, blood) from the nanocomponents, including viruses. To clarify the idea, let us consider a system consisting of the metal surface at which the regular very long cylinders are situated. At this complicated surface, the surface plasmon-polaritons can be excited (Fig. 1). Due to interaction between the localized near the cylinders plasmons and surface plasmon-polaritons, the very complicated distribution of local field (along the OX) will be formed. This localfield distribution is schematically shown by the insert in Fig. 1. If one would suppose that some small nanoparticles, the linear dimensions of which are close to or smaller than the diameter of these cylinders, will be adsorbed at the surface, one should take into account that adsorption will occur at the surface with periodically changed local field. Then, it can be realized the situation when the nanoparticles will adsorbed at the surface mainly within domains of strong local field. The interaction potential (its attractive part) between the nanoparticle and surface can be evaluated as [8] 
$U(\mathbf{r}, l)=-\frac{\hbar}{4 \pi^{2}} \int_{0}^{\infty} d \omega \operatorname{cth}(\hbar \omega / 2 k T) \times$

$\times \operatorname{Im}\left[\chi_{i j}^{(p)}(\omega) I_{i j}(0, l, l, \omega) e^{-i \mathbf{k r}}\right]$,

where $\chi_{i j}^{(p)}(\omega)$ is the effective susceptibility of the single nanoparticle, $I_{i j}\left(\mathbf{r}-\mathbf{r}^{\prime}, l, l, \omega\right)$ - indirect part of electrodynamics Green function for the substrate, and $\mathbf{k}$ - wave vector of the surface periodic structure. Then, supposing that the nanoparticles are shaped as ellipsoidal particles, the effective susceptibility $\chi_{i j}^{(p)}(\omega)$ can be written in the form $[9,10]$

$X_{i j}(\omega)=\chi(\omega) \frac{1}{\varepsilon_{m}+\chi(\omega) L_{i}} \delta_{i j}$,

where

$L_{i}=\frac{a_{x} a_{y} a_{z}}{2} \int_{0}^{\infty} \frac{d s}{\left(s+a_{i}^{2}\right) \sqrt{\left(s+a_{x}^{2}\right)\left(s+a_{y}^{2}\right)\left(s+a_{z}^{2}\right)}}$,

are depolarization factors, $a_{x}, a_{y}, a_{z}$ mean the semi-axes of ellipsoid, $\varepsilon_{m}$ is the dielectric permittivity of the medium where the particle is embedded. The electrodynamics properties of the surface with the periodic structure can be described with the Green function $I_{i j}\left(\mathbf{r}-\mathbf{r}^{\prime}, l, l, \omega\right)$. The Green function in the socalled k-p representation can be written in the form [11] $I_{l k}\left(\mathbf{k}, z, z^{\prime}, \omega\right)=L_{l j}(\mathbf{k}, \mathbf{k} \pm \mathbf{G}, z, \omega) G_{j k}^{(0)}\left(\mathbf{k}, d, z^{\prime}, \omega\right)$, where $G_{j k}^{(0)}\left(\mathbf{k}, z, z^{\prime}, \omega\right)$ is the electrodynamics Green function of the upper semi-infinite medium, and $L_{l j}(\mathbf{k}, \mathbf{k}, \pm \mathbf{G}, z, \omega)$ is the local-field factor [3]. The localfield factor has the form

$L_{l j}(\mathbf{k}, \mathbf{k}, \pm \mathbf{G}, z, \omega)=\Omega_{l j}^{-1}(\mathbf{k}, \mathbf{k}, \pm \mathbf{G}, z, \omega)$,

$\Omega_{l j}^{-1}(\mathbf{k}, \mathbf{k}, \pm \mathbf{G}, z, \omega)=\left[\varphi_{j l}^{-1}(\mathbf{k}, z, \omega)-a^{2} n^{2} G_{j l}(\mathbf{k}, z, \omega) \times\right.$

$\times \chi_{i l^{\prime}}^{(+)}(\omega) \varphi_{l^{\prime} m}(\mathbf{k}+G, z, \omega) G_{m i^{\prime}}(\mathbf{k}+G, z, \omega) \chi_{i^{\prime} l}^{(-)}(\omega)-$

$-a^{2} n^{2} G_{j l}(\mathbf{k}, z, \omega) \chi_{i l^{\prime}}^{(-)}(\omega) \varphi_{l^{\prime} m}(\mathbf{k}-G, z, \omega) \times$

$\left.\times G_{m i^{\prime}}(\mathbf{k}-G, z, \omega) \chi_{i^{\prime} l}^{(-)}(\omega)\right]$.

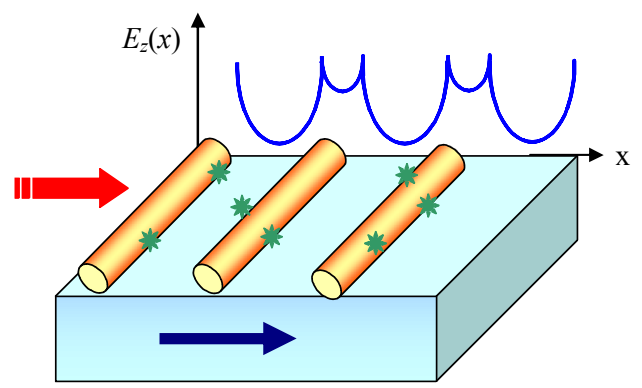

Fig. 1. Sketch of the system under consideration. The line qualitatively shows a spatial distribution of the interaction potential along the $x$-axis.
The next designations were used here: $\mathbf{G}$ is a smallest vector of the reciprocal 2D lattice,

$\varphi_{n k}(\mathbf{k} \pm \mathbf{G}, z, \omega)=\left[\delta_{k n}-a n G_{k j}(\mathbf{k} \pm \mathbf{G}, z, \omega) \chi_{j n}^{(0)}(\omega)\right]^{-1}$

$\varphi_{n k}(\mathbf{k}, z, \omega)=\left[\delta_{k n}-a n G_{k j}(\mathbf{k}, z, \omega) \chi_{j n}^{(0)}(\omega)\right]^{-1}$,

$\int_{V} d \mathbf{r} \chi_{j l}(\mathbf{r}, \omega)=\chi_{j l}^{(0)}(\omega)$

and

$\int_{V} d \mathbf{r} e^{ \pm i \mathbf{G r}} \chi_{j l}(\mathbf{r}, \omega)=\chi_{j l}^{( \pm)}(\omega)$ are the averaged effective susceptibilities of the nanocylinders, $\chi_{j l}(\mathbf{r}, \omega)$ is the effective susceptibility of a single nanocylinder at the surface which dielectric properties are written by the Green function $G_{i j}\left(\mathbf{k}, z, z^{\prime}, \omega\right)$. All the Green functions $G_{i j}\left(\mathbf{k}, z, z^{\prime}, \omega\right)$ in Eqs. (5-7) are taken with $z^{\prime}=d$, where $d$ is the radius of the nanocylinder. As the local-field factor $L_{l j}(\mathbf{k}, \mathbf{k} \pm \mathbf{G}, z, \omega)$ relates the local field $E_{i}(\mathbf{k}, z, \omega)$ and external long-range field $E_{i}^{(0)}(\mathbf{k}, z, \omega)$, one can see that the strong local-field domains are determined by high values of local-field factor. Then, from Eqs. (1) and (4) one can see that domains of strong local fields correspond to the strong interaction energy between the nanoparticle and surface. The surface wave excitation and propagation means that the real part of determinant for the matrix $\Omega_{l j}(\mathbf{k}, \mathbf{k} \pm \mathbf{G}, z, \omega)$ becomes equal to zero. So, it should be expected that small nanoparticles will be better adsorbed at the surface domain of the strong local field. On the other hand, it is well known that the strong local fields are formed at the edge of the nano-stripe structures at the surface, along which the surface electromagnetic waves propagate $[12,13]$. If the nanostripes are situated alongside each other and not so far (several tens of nanometers), the small nanoparticles (like viruses) can be adsorbed at the edges of the stripes (at the edges of the nanocylinders). If one assumes that the blood, in which for instance AIDS viruses are presented, flows along the surface shown in Fig. 1, these viruses can be adsorbed by the structured surface, but blood organelles having larger linear dimensions could not be efficiently adsorbed at this surface.

\section{References}

1. K. Zimmer, A Planet of Viruses. University of Chicago Press, 2011.

2. D.H. Crowford, Viruses: A Very Short Introduction. Oxford University Press, 2011.

3. O. Keller, Local fields in the electrodynamics of mesoscopic media // Phys. Repts. 268, p. 82 (1996). 
4. Ch. Girard, Ch. Joachim, S. Gauthier, The physics of the near filed // Repts. Progr. Phys. 63, p. 893 (2000).

5. K.-H. Dostert, M. Álvarez, K. Koynov, A. del Campo, H.-J. Butt, and M. Kreiter, Near field guided chemical nanopattering // Langmuir, 28, p. 3699 (2012).

6. J. Zhang, J. Yang, X. Wu and Q. Gong, Electric field enhancing properties of the V-shaped optical resonant antennas // Opt. Express, 15, p. 16852 (2007).

7. Ming-Yaw Ng, Wei-Chih Liu, Local-field confinement in three-pair arrays of metallic nanocylinders // Opt. Express, 14, p. 4504 (2006).

8. Yu. Barash and V. Ginzburg, Some problems of the theory of Van der Waals forces // Uspekhi fiz. nauk, 27, p. 467 (1984), in Russian.
9. L.D. Landau, E.M. Lifshitz, and L.P. Pitaevskii, Electrodynamics of Continuous Media. Butterworth-Heinemann, 1984.

10. V. Lozovski, A. Tsykhonya, Dispersion properties of nano-scale systems // Intern. J. Theor. Phys., Group Theory and Nonlinear Optics, 12, p. 31 (2007).

11. V. Lozovski, S. Shrader, A. Tsykhonya, Possibility of surface plasmon-polaritons amplification by direct current in two-interface systems with 2D Bragg structure on the surface // Opt. Communs. 282, p. 3257 (2009).

12. V.A. Sterligov, Ya. Men, P.M. Lytvyn, Giant enhancement of elastic surface plasmon-polariton scattering // Opt. Express, 18, p. 43 (2010).

13. T.A. Leskova, A.A. Maradudin, W. Zierau, Surface plasmon polariton propagation near an index step // Opt. Communs. 249, p. 23 (2005). 